\title{
Prevention of alcohol withdrawal seizure recurrence and treatment of other alcohol withdrawal symptoms in the emergency department: a rapid review
}

\author{
Justin Jek-Kahn Koh ${ }^{1,2^{*}}$ (D) Madeline Malczewska ${ }^{3}$, Mary M. Doyle-Waters ${ }^{4}$ and Jessica Moe ${ }^{5,6,7}$
}

\begin{abstract}
Background: Patients who experience harms from alcohol and other substance use often seek care in the emergency department (ED). ED visits related to alcohol withdrawal have increased across the world during the COVID-19 pandemic. ED clinicians are responsible for risk-stratifying patients under time and resource constraints and must reliably identify those who are safe for outpatient management versus those who require more intensive levels of care. Published guidelines for alcohol withdrawal are largely limited to the primary care and outpatient settings, and do not provide specific guidance for ED use. The purpose of this review was to synthesize published evidence on the treatment of alcohol withdrawal syndrome in the ED.
\end{abstract}

Methods: We conducted a rapid review by searching MEDLINE, Embase, and the Cochrane Central Register of Controlled Trials (1980 to 2020). We searched for grey literature on Google and hand-searched the conference abstracts of relevant addiction medicine and emergency medicine professional associations (2015 to 2020). We included interventional and observational studies that reported outcomes of clinical interventions aimed at treating alcohol withdrawal syndrome in adults in the ED.

Results: We identified 13 studies that met inclusion criteria for our review (7 randomized controlled trials and 6 observational studies). Most studies were at high/serious risk of bias. We divided studies based on intervention and summarized evidence narratively. Benzodiazepines decrease alcohol withdrawal seizure recurrence and treat other alcohol withdrawal symptoms, but no clear evidence supports the use of one benzodiazepine over another. It is unclear if symptom-triggered benzodiazepine protocols are effective for use in the ED. More evidence is needed to determine if phenobarbital, with or without benzodiazepines, can be used safely and effectively to treat alcohol withdrawal in the ED. Phenytoin does not have evidence of effectiveness at preventing withdrawal seizures in the ED.

\footnotetext{
* Correspondence: justin.koh@usask.ca

${ }^{1}$ Addiction Medicine Fellowship Program, British Columbia Centre for

Substance Use, Vancouver, BC, Canada

${ }^{2}$ Royal College Emergency Medicine Residency Program, Department of

Emergency Medicine, College of Medicine, University of Saskatchewan,

Saskatoon, SK, Canada

Full list of author information is available at the end of the article
}

(c) The Author(s). 2021 Open Access This article is licensed under a Creative Commons Attribution 4.0 International License, which permits use, sharing, adaptation, distribution and reproduction in any medium or format, as long as you give appropriate credit to the original author(s) and the source, provide a link to the Creative Commons licence, and indicate if changes were made. The images or other third party material in this article are included in the article's Creative Commons licence, unless indicated otherwise in a credit line to the material. If material is not included in the article's Creative Commons licence and your intended use is not permitted by statutory regulation or exceeds the permitted use, you will need to obtain permission directly from the copyright holder. To view a copy of this licence, visit http://creativecommons.org/licenses/by/4.0/ The Creative Commons Public Domain Dedication waiver (http://creativecommons.org/publicdomain/zero/1.0/) applies to the data made available in this article, unless otherwise stated in a credit line to the data. 
Conclusions: Few studies have evaluated the safety and efficacy of pharmacotherapies for alcohol withdrawal specifically in the ED setting. Benzodiazepines are the most evidence-based treatment for alcohol withdrawal in the ED.

Pharmacotherapies that have demonstrated benefit for treatment of alcohol withdrawal in other inpatient and outpatient settings should be evaluated in the ED setting before routine use.

Keywords: Alcohol, Substance use, Emergency department, Drug therapy

\section{Background}

Rationale

Patients who experience harms from alcohol and other substance use often seek care in the emergency department (ED) $[1,2]$. In recent years, ED visits related to alcohol in North America have increased significantly [3, 4]. In the United States, the rate of acute alcohol-related ED visits increased 40\% between 2006 and 2014. Although national-level data are lacking in Canada, a provincial-level analysis in Ontario demonstrated that between 2003 to 2016, the increase in rates of alcoholrelated visits was 4.4 times greater than the increase in all-cause ED visits [3].

In 2016, alcohol use was the seventh-leading risk factor for deaths and disability-adjusted life years globally [5]. Among heavy alcohol users admitted for hospital management, the incidence of alcohol withdrawal syndrome is estimated to be 1.9 to $6.7 \%$ [6]. Many patients with alcohol use disorder will require management of alcohol withdrawal during their ED visit $[7,8]$. Alcohol withdrawal in the ED has been associated with increased use of critical care resources [9], and frequent ED visits for alcoholrelated presentations have been associated with high relative mortality rates $[1,10]$. Furthermore, recent studies have demonstrated significant increases in the proportion of alcohol withdrawal-related ED visits during the COVID-19 pandemic, highlighting the rapidly growing burden of alcohol use on EDs across the world [11-14].

ED clinicians are responsible for risk-stratifying patients with alcohol withdrawal syndrome under time and resource constraints, and must reliably identify those who are safe for outpatient management versus those who require more intensive levels of care [7]. Published clinical guidelines recommend stratifying patients with alcohol withdrawal based on their risk of developing complications (e.g., generalized tonic-clonic seizures and delirium tremens) [15-18]. These guidelines are largely limited to the primary care and outpatient settings and do not provide specific guidance for ED clinicians [15-17].

Although one recent literature review summarized evidence for ED withdrawal management, the authors extrapolated recommendations from guidelines for nonED settings [19]. Another recent literature review also included evidence from non-ED studies [20]. Given the ongoing rise in ED visits due to alcohol withdrawal, and further exacerbation during the current COVID-19 pandemic, there is an urgent need to determine whether certain clinical interventions, when initiated in the ED, could reduce the need for admission and/or prevent other complications of alcohol withdrawal.

\section{Objectives}

Our main objective was to synthesize evidence from published studies on the treatment of alcohol withdrawal syndrome among adult ED patients.

\section{Methods}

In this rapid review, we adapted traditional systematic review methods to generate evidence within an accelerated time frame [21-23]. Rapid reviews are a pragmatic and resource-efficient approach to knowledge synthesis that remains scientific, transparent and reproducible [24]. The utility and importance of rapid reviews is recognized by the Cochrane Rapid Review Methods Group [25], and health policy institutions such as the World Health Organization and the Canadian Agency for Drugs and Technologies in Health [26, 27].

We prepared this paper in accordance with the 2009 Preferred Reporting Items for Systematic Reviews and Meta-Analyses (PRISMA) checklist [28]. Our adaptations to allow rapid review were that one reviewer performed title/abstract screening and quality assessments rather than two independent reviewers performing these steps in duplicate. Our search strategy utilized focused search terms in the most highly relevant databases to prioritize yielding citations with greatest relevance.

\section{Protocol and registration}

In line with our goal of producing this evidence summary expeditiously, we did not publish a review protocol or register this review prior to study initiation.

\section{Eligibility criteria Population}

Adult patients (18 years and older) who presented to the ED with any clinical feature of alcohol withdrawal syndrome, as determined by criteria specified by study authors, e.g., Clinical Institute Withdrawal Assessment for Alcohol (CIWA) score. 


\section{Intervention}

Any clinical intervention aimed at treating alcohol withdrawal symptoms, signs, or complications; and administered via any route. We excluded studies that examined psychosocial interventions alone, or supportive interventions alone.

\section{Outcome}

Any clinical or patient-oriented outcome related to alcohol withdrawal.

\section{Study design}

Interventional studies with or without a comparator group, including randomized controlled trials (RCT) and non-randomized trials, as well as observational cohort studies that evaluated an intervention. We excluded review articles and case reports, studies published prior to 1980, non-English publications, and non-human studies.

\section{Information sources}

We searched MEDLINE, Embase, and Cochrane Central Register of Controlled Trials (CENTRAL) from 1980 to 2020 through Ovid. All three databases were last searched on May 11, 2020.

A professional health sciences librarian (MDW) developed our search strategy. We searched MEDLINE and CENTRAL using concepts emergency department AND alcohol withdrawal AND (drugs OR drug subheadings). We searched EMBASE using concepts emergency department AND alcohol withdrawal (focused) AND (drugs OR drug subheadings), as well as concepts emergency department AND alcohol withdrawal AND drugs AND drug subheadings. Subheadings and keywords were included in the searches to increase sensitivity.

We performed the grey literature search using the search engine Google using combinations of terms ["emergency department", "emergency room", "emergency" or "accident and emergency"] and ["alcohol withdrawal"] and ["treatment" or "intervention" or "management"]. The first 50 search results were opened and reviewed for relevant materials. We also hand-searched the most recent conference abstracts (2015 to 2020) of the Canadian Society of Addiction Medicine, American Society of Addiction Medicine, Canadian Association of Emergency Physicians, and American College of Emergency Physicians.

\section{Search}

We report our full electronic search strategy for MEDLINE (Ovid) in Appendix 1.

\section{Study selection}

A single reviewer (MM) performed title and abstract screening. Before full-text screening, articles were flagged for secondary review by the principal investigator
(JM) as needed. Inclusion and exclusion decisions for full-text articles were performed in duplicate by two trained reviewers (MM and JK).

\section{Data collection process}

Data extraction was performed independently and in duplicate by two extractors (MM and JK). The principal investigator (JM) arbitrated and resolved any issues that arose during data extraction.

\section{Data items}

We extracted information relating to the study design and characteristics, and results as follows:

\section{Study characteristics}

authors, year of publication, study design, study location, study time period (start and end dates), follow-up period (if applicable), data sources.

\section{Study participants}

inclusion and exclusion criteria, age, sex, ethnicity, alcohol withdrawal severity at presentation, method of determining alcohol withdrawal, comorbidities, number of participants in main analysis, losses to follow-up.

\section{Study intervention}

method of allocation, method of determining eligibility for intervention, description of intervention (type, duration, dose, and timing), person administering intervention, other components of the intervention, method of determining end-point, components of the intervention after ED visit, follow-up after ED visit.

\section{Study outcomes}

person ascertaining outcomes, primary outcomes, secondary and tertiary outcomes, adverse events.

\section{Risk of bias in individual studies}

We used the Cochrane risk-of-bias tool for randomized trials Version 2 (RoB 2) to assess the risk of bias in the RCTs included in this study [29]. For non-randomized studies, we used the Cochrane risk of bias in nonrandomized studies of interventions (ROBINS-I) tool [30]. Risk of bias assessments were performed by one trained reviewer $(\mathrm{JK})$ and verified by the principal investigator (JM).

\section{Synthesis of results}

Due to clinical and methodological heterogeneity of included RCTs, we did not meta-analyze their results. Instead, we present a narrative summary of the results of all included studies. 


\section{Results}

\section{Study selection}

Our search retrieved a total of 214 references after 46 duplicates were removed from searches in health databases. Two other papers were found through grey literature searches. Following title/abstract and full-text inclusion screens, we identified 13 studies that met inclusion criteria for our review. The study flow diagram is displayed in Fig. 1.

\section{Study characteristics}

There were seven RCTs, two retrospective cohort studies, and four retrospective chart reviews. Publication dates ranged from 1989 to 2020. Study characteristics are displayed in Table 1.

\section{Risk of bias within studies}

Among the seven RCTs, one was at high risk of bias, two had some concerns, and the remainder was at low risk. All non-randomized studies were at serious risk of bias. Table 2 lists the risk of bias assessments for the studies we summarized.

\section{Results of individual studies}

Table 2 also lists the main outcomes and key findings for the studies we summarized. We reported effect sizes whenever available.

\section{Synthesis of results \\ Benzodiazepines alone}

Benzodiazepines vs. placebo Two RCTs published before 2000 compared the use of benzodiazepines vs. placebo [31, 39]. One RCT $(n=186)$ of adults with witnessed generalized seizures found that normal saline placebo resulted in a significantly higher risk of recurrent seizure within six hours when compared to a single $2 \mathrm{mg}$ dose of IV lorazepam (odds ratio 10.4, 95\% CI: 3.6 to 30.2) [31]. Another RCT $(n=41)$ of patients in mildto-moderate alcohol withdrawal (without medical complications or witnessed seizures) reported greater proportions of patients with improvement in withdrawal scores (defined as CIWA score $\leq 10$ ) between groups who were allocated to sublingual lorazepam compared to sublingual placebo (95.2\% vs. $85.0 \%, p<0.001)$ [39].

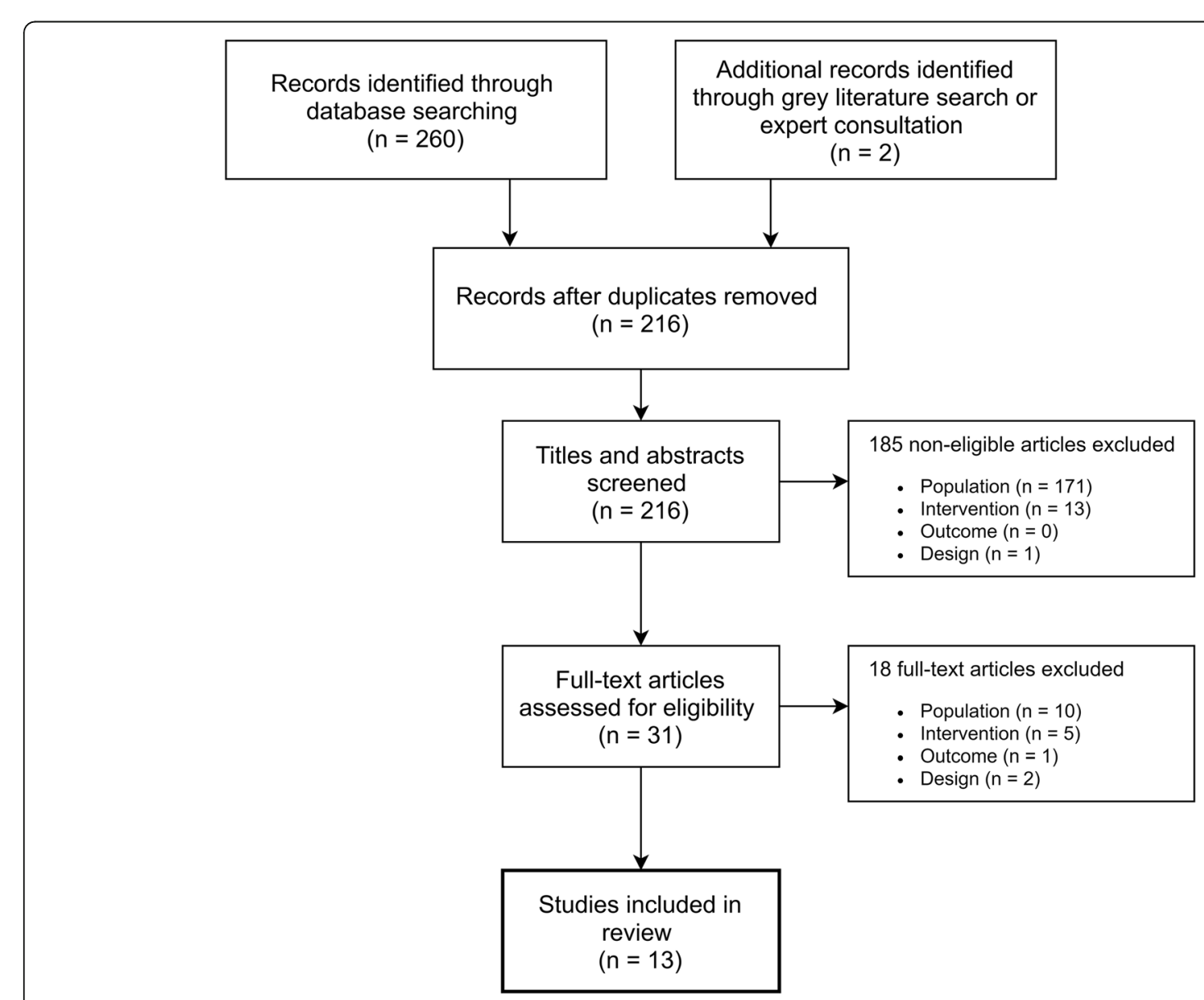

Fig. 1 Evidence search and selection 
Table 1 Study characteristics (grouped by intervention)

\begin{tabular}{|c|c|c|c|c|}
\hline Author & Year & Location & Study design & $N$ \\
\hline \multicolumn{5}{|l|}{ Benzodiazepines alone } \\
\hline \multicolumn{5}{|l|}{ Benzodiazepines vs. placebo } \\
\hline D’Onofrio G, Rathlev NK, Ulrich AS, Fish SS, Freedland ES [31] & 1999 & United States & Randomized controlled trial & 186 \\
\hline Naranjo CA, Sellers EM, Chater K, Iversen P, Roach C, Sykora K [32] & 1983 & Canada & Randomized controlled trial & 41 \\
\hline \multicolumn{5}{|l|}{ Benzodiazepine selection } \\
\hline Scheuermeyer FX, Miles I, Lane DJ, Grunau B, Grafstein E, Sljivic I, et al. [32] & 2020 & Canada & Retrospective chart review & 898 \\
\hline \multicolumn{5}{|l|}{ Symptom-triggered therapy } \\
\hline Ismail MF, Doherty K, Bradshaw P, O'Sullivan I, Cassidy EM [33] & 2019 & Ireland & Retrospective chart review & 174 \\
\hline Cassidy EM, O'Sullivan I, Bradshaw P, Islam T, Onovo C [35] & 2012 & Ireland & Retrospective chart review & 99 \\
\hline \multicolumn{5}{|l|}{ Combined phenobarbital and benzodiazepines } \\
\hline Ibarra Jr. F [36] & 2020 & United States & Retrospective chart review & 78 \\
\hline Sullivan SM, Dewey BN, Jarrell DJ, Vadiei N, Patanwala AE [34] & 2019 & United States & Retrospective cohort study & 209 \\
\hline Rosenson J, Clements C, Simon B, Vieaux J, Graffman S, Vahidnia F, et al. [35] & 2013 & United States & Randomized controlled trial & 198 \\
\hline \multicolumn{5}{|l|}{ Phenobarbital alone } \\
\hline Nelson AC, Kehoe J, Sankoff J, Mintzer D, Taub J, Kaucher KA [36] & 2019 & United States & Retrospective cohort study & 300 \\
\hline Hendey GW, Dery RA, Barnes RL, Snowden B, Mentler P [40] & 2011 & United States & Randomized controlled trial & 44 \\
\hline \multicolumn{5}{|l|}{ Phenytoin alone } \\
\hline Rathlev NK, D'Onofrio G, Fish SS, Harrison PM, Bernstein E, Hossack RW, et al. [37] & 1994 & United States & Randomized controlled trial & 100 \\
\hline Chance JF [42] & 1991 & United States & Randomized controlled trial & 55 \\
\hline Alldredge BK, Lowenstein DH, Simon RP [38] & 1989 & United States & Randomized controlled trial & 90 \\
\hline
\end{tabular}

Benzodiazepine selection One retrospective chart review $(n=898)$ conducted in multiple EDs in Vancouver, Canada compared patients who received lorazepam versus diazepam as their initial management in the ED. [32] Initial CIWA scores were similar between groups (median 17 [IQR 13 to 22]). They reported no differences in terms of hospital admission ( $-1.2,95 \% \mathrm{CI}:-6.3$ to 4.2 ). Of note, physicians in the study were free to select their choice of benzodiazepine and route of administration.

Symptom-triggered therapy Two studies reported results of a symptom-triggered protocol implemented in an ED clinical decision unit [33, 40]. One descriptive retrospective chart review $(n=174)$ reported that patients placed on a symptom-triggered benzodiazepine protocol received a median cumulative diazepam dose of $20 \mathrm{mg}$ (IQR $80 \mathrm{mg}$ ), and $97.1 \%$ were ultimately discharged from the ED, although they did not have a comparison group [33]. Another retrospective chart review $(n=99)$ compared those placed on a CIWA-based symptom-triggered protocol with a non-matched comparison group that received a standard tapered benzodiazepine regime [40]. The symptom-triggered group received lower cumulative benzodiazepine doses (median $80 \mathrm{mg}$ vs. $170 \mathrm{mg}, p=0.000$ ), and had shorter lengths of stay (median 2 days vs. 3 days, $p=0.006$ ).

\section{Combined phenobarbital and benzodiazepines}

In one retrospective chart review $(n=78)$, patients who received a single IV dose of phenobarbital (130 to $260 \mathrm{mg}$ ) in the ED with symptom-triggered lorazepam compared to those who only received symptom-triggered lorazepam had no statistically significant differences in terms of ED discharges $(10.0 \%$ vs. $5.3 \%, p=0.43)$, hospital admissions (85.0\% vs. $84.2 \%, p=0.92)$, or intensive care unit (ICU) admissions (5.0\% vs. $10.5 \%, p=0.36)$ [41].

In another retrospective cohort study $(n=209)$, the phenobarbital and benzodiazepine groups had similar proportions of ICU admission (14.4\% vs. $10.7 \%, p=0.53)$, ED length of stay ( $9 \mathrm{~h}$ vs $9 \mathrm{~h}, p=0.048$ ), and CIWA scores at ED discharge ( 7 vs. $7, p=0.32$ ) [34]. Of note, $81 \%$ of the phenobarbital group also received benzodiazepines. The two groups did not differ significantly in terms of complications, such as intubation and seizure.

Finally, one RCT $(n=198)$ compared a single dose of phenobarbital $(10 \mathrm{mg} / \mathrm{kg}$ IV over $30 \mathrm{~min}$ ) followed by symptom-triggered lorazepam (oral/IV) versus symptom-triggered lorazepam only [35]. The phenobarbital group had significantly fewer ICU admissions than the placebo group (7.8\% vs. $25.5 \%$, difference $17 \%$ [95\% CI: 4-32]), but there was no difference in adverse outcomes (intubation, seizure, use of mechanical restraints, and need for bedside sitter). 
Table 2 Main outcomes, key findings, and risk of bias assessments for summarized studies

\begin{tabular}{|c|c|c|c|c|c|c|c|}
\hline Author & Participants & Intervention & Comparison & $\begin{array}{l}\text { Main } \\
\text { outcome(s) }\end{array}$ & Key results & & $\begin{array}{l}\text { Risk of } \\
\text { bias }\end{array}$ \\
\hline $\begin{array}{l}\text { Benzodiazepines } \\
\text { alone }\end{array}$ & & & & & $\begin{array}{l}\text { Intervention } \\
\text { group }\end{array}$ & Comparison group & \\
\hline \multicolumn{8}{|c|}{ Benzodiazepine vs. placebo } \\
\hline $\begin{array}{l}\text { D'Onofrio G, } \\
\text { Rathlev NK, } \\
\text { Ulrich AS, Fish } \\
\text { SS, Freedland } \\
\text { ES (1999) [31] }\end{array}$ & $\begin{array}{l}\text { Adults with } \\
\text { witnessed } \\
\text { generalized seizure } \\
\text { related to alcohol }\end{array}$ & $\begin{array}{l}\text { Lorazepam } 2 \text { mg IV } \\
(n=100)\end{array}$ & Placebo $(n=86)$ & $\begin{array}{l}\text { Seizure } \\
\text { recurrence } \\
\text { within } 6 \mathrm{~h} \text { of } \\
\text { intervention }\end{array}$ & $\begin{array}{l}3 / 100(3.0 \%) \\
*\end{array}$ & $\begin{array}{l}\text { 21/86 ( } 24.4 \%) \text { odds } \\
\text { ratio } 10.4 \\
\text { (95\% Cl: } 3.6 \text { to } 30.2 \text { ) }\end{array}$ & Low \\
\hline $\begin{array}{l}\text { Naranjo CA, } \\
\text { Sellers EM, } \\
\text { Chater K, } \\
\text { Iversen P, } \\
\text { Roach C, } \\
\text { Sykora K (1983) } \\
\text { [39] }\end{array}$ & $\begin{array}{l}\text { Adults with mild-to- } \\
\text { moderate AWS } \\
\text { (clinician } \\
\text { assessment) }\end{array}$ & $\begin{array}{l}\text { Scheduled } \\
\text { lorazepam } 2 \text { mg PO } \\
\text { q2h (max. } 3 \text { doses) } \\
(n=21)\end{array}$ & Placebo $(n=20)$ & $\begin{array}{l}\text { Seizure } \\
\text { recurrence } \\
\text { within } 6 \mathrm{~h} \text { of } \\
\text { intervention }\end{array}$ & $1 / 21(4.8 \%) *$ & $3 / 20(15.0 \%)$ & Low \\
\hline \multicolumn{8}{|c|}{ Benzodiazepine selection } \\
\hline \multirow{3}{*}{$\begin{array}{l}\text { Scheuermeyer } \\
\text { FX, Miles I, } \\
\text { Lane DJ, } \\
\text { Grunau B, } \\
\text { Grafstein E, } \\
\text { Slijicic l, et al. } \\
\text { (2020) [32] }\end{array}$} & \multirow[t]{3}{*}{$\begin{array}{l}\text { Adults with AWS } \\
\text { (ED discharge } \\
\text { diagnosis) treated } \\
\text { with lorazepam or } \\
\text { diazepam }\end{array}$} & \multirow[t]{3}{*}{$\begin{array}{l}\text { Lorazepam (dose } \\
\text { and route at } \\
\text { physician } \\
\text { discretion) } \\
(n=394)\end{array}$} & \multirow[t]{3}{*}{$\begin{array}{l}\text { Diazepam (dose } \\
\text { and route at } \\
\text { physician } \\
\text { discretion) } \\
(n=504)\end{array}$} & $\begin{array}{l}\text { 1. Hospital } \\
\text { admission } \\
\text { (including ICU) }\end{array}$ & $\begin{array}{l}69 / 394 \\
(17.5 \%) \text { effect } \\
\text { size: }-1.2 \\
(95 \% \mathrm{Cl}:- \\
6.3 \text { to } 4.2)\end{array}$ & $94 / 504(18.7 \%)$ & \multirow[t]{3}{*}{ Serious } \\
\hline & & & & $\begin{array}{l}\text { 2. Seizures in ED } \\
\text { before } \\
\text { treatment }\end{array}$ & $\begin{array}{l}\text { 3/394 (0.8\%) } \\
\text { *effect size: } \\
-0.03 \\
(95 \% \mathrm{Cl}:-1.7 \\
\text { to } 1.5)\end{array}$ & $4 / 504(0.8 \%)$ & \\
\hline & & & & $\begin{array}{l}\text { 3. ED length of } \\
\text { stay (non- } \\
\text { admitted } \\
\text { patients) }\end{array}$ & $\begin{array}{l}\text { Median } 266 \\
\text { min } \\
\text { (IQR } 163 \text { to } \\
387) \text { effect } \\
\text { size: }-33 \\
(95 \% \text { Cl: }-75 \\
\text { to }-6)\end{array}$ & $\begin{array}{l}\text { Median } 299 \mathrm{~min} \\
\text { (IQR } 192 \text { to 463) }\end{array}$ & \\
\hline \multicolumn{8}{|c|}{ Symptom-triggered therapy } \\
\hline \multirow{4}{*}{$\begin{array}{l}\text { Ismail MF, } \\
\text { Doherty K, } \\
\text { Bradshaw P, } \\
\text { O'Sullivan I, } \\
\text { Cassidy EM } \\
\text { (2019) [33] }\end{array}$} & \multirow{4}{*}{$\begin{array}{l}\text { Adults with AWS } \\
\text { (clinician } \\
\text { assessment) placed } \\
\text { on treatment } \\
\text { protocol in a short } \\
\text { stay clinical decision } \\
\text { unit }\end{array}$} & \multirow{4}{*}{$\begin{array}{l}\text { Symptom-triggered } \\
\text { diazepam (route } \\
\text { not specified) } \\
\text { when CIWA } \geq 10 \\
(n=174)\end{array}$} & \multirow[t]{4}{*}{ N/A } & $\begin{array}{l}\text { 1. Cumulative } \\
\text { diazepam dose }\end{array}$ & $\begin{array}{l}\text { Median 20 } \\
\text { mg (IQR 80) }\end{array}$ & \multirow[t]{4}{*}{ N/A } & \multirow[t]{4}{*}{ Serious } \\
\hline & & & & $\begin{array}{l}\text { 2. Duration of } \\
\text { symptom- } \\
\text { triggered } \\
\text { protocol }\end{array}$ & $\begin{array}{l}\text { Median } 12 \mathrm{~h} \\
(\mathrm{IQR} 12)\end{array}$ & & \\
\hline & & & & $\begin{array}{l}\text { 3. Length of } \\
\text { stay in clinical } \\
\text { decision unit }\end{array}$ & $\begin{array}{l}\text { Median } 22 \text { h } \\
(\text { IQR 20) }\end{array}$ & & \\
\hline & & & & 4. ED discharge & $\begin{array}{l}169 / 174 \\
(97.1 \%)\end{array}$ & & \\
\hline \multirow[t]{2}{*}{$\begin{array}{l}\text { Cassidy EM, } \\
\text { O'Sullivan I, } \\
\text { Bradshaw P, } \\
\text { Islam T, Onovo } \\
\text { C (2012) [40] }\end{array}$} & \multirow[t]{2}{*}{$\begin{array}{l}\text { Adults with AWS } \\
\text { (clinician } \\
\text { assessment) treated } \\
\text { in the ED clinical } \\
\text { decision unit }\end{array}$} & \multirow[t]{2}{*}{$\begin{array}{l}\text { Symptom-triggered } \\
\text { benzodiazepine } \\
(n=49)\end{array}$} & \multirow[t]{2}{*}{$\begin{array}{l}\text { Fixed dose } \\
\text { benzodiazepine } \\
(n=50)\end{array}$} & $\begin{array}{l}\text { 1. Cumulative } \\
\text { benzodiazepine } \\
\text { dose (in } \\
\text { diazepam } \\
\text { equivalents) }\end{array}$ & $\begin{array}{l}\text { Median } 80 \\
\text { mg } \\
\text { (range } 0 \text { to } \\
900)^{*}\end{array}$ & $\begin{array}{l}\text { Median } 170 \mathrm{mg} \\
\text { (range } 15 \text { to } 720 \text { ) }\end{array}$ & \multirow[t]{2}{*}{ Serious } \\
\hline & & & & $\begin{array}{l}\text { 2. Hospital } \\
\text { length of stay }\end{array}$ & $\begin{array}{l}\text { Median } 2 \\
\text { days } \\
\text { (range } 1 \text { to } \\
\text { 9) * }\end{array}$ & $\begin{array}{l}\text { Median } 3 \text { days } \\
\text { (range } 1 \text { to 12) }\end{array}$ & \\
\hline \multicolumn{8}{|c|}{ Combined phenobarbital and benzodiazepines } \\
\hline $\begin{array}{l}\text { Ibarra Jr. F } \\
\text { (2020) [41] }\end{array}$ & $\begin{array}{l}\text { Adults with } \\
\text { moderate/severe } \\
\text { AWS requiring }\end{array}$ & $\begin{array}{l}\text { Phenobarbital } 130 \\
\text { to } 260 \mathrm{mg} \mathrm{IV} \mathrm{+} \\
\text { symptom-triggered }\end{array}$ & $\begin{array}{l}\text { Symptom- } \\
\text { triggered } \\
\text { lorazepam PO/IV }\end{array}$ & $\begin{array}{l}\text { 1. Total } \\
\text { lorazepam } \\
\text { doses (Day 1) }\end{array}$ & $\begin{array}{l}\text { Median } 16 \\
\text { mg } \\
\text { (IQR } 6 \text { to } 32 \text { ) }\end{array}$ & $\begin{array}{l}\text { Median } 10 \mathrm{mg} \\
\text { (IQR } 6 \text { to } 19 \text { ) }\end{array}$ & Serious \\
\hline
\end{tabular}


Table 2 Main outcomes, key findings, and risk of bias assessments for summarized studies (Continued)

\begin{tabular}{|c|c|c|c|c|c|c|c|}
\hline Author & Participants & Intervention & Comparison & $\begin{array}{l}\text { Main } \\
\text { outcome(s) }\end{array}$ & Key results & & $\begin{array}{l}\text { Risk of } \\
\text { bias }\end{array}$ \\
\hline & $\begin{array}{l}\text { treatment (clinician } \\
\text { assessment) }\end{array}$ & $\begin{array}{l}\text { lorazepam PO/IV } \\
(n=40)\end{array}$ & $(n=38)$ & $\begin{array}{l}\text { 2. Total } \\
\text { lorazepam } \\
\text { doses (Day 2) }\end{array}$ & $\begin{array}{l}\text { Median } 10 \\
\text { mg } \\
\text { (IQR } 2 \text { to 29) }\end{array}$ & $\begin{array}{l}\text { Median } 6 \text { mg } \\
\text { (IQR } 2 \text { to } 12 \text { ) }\end{array}$ & \\
\hline & & & & $\begin{array}{l}\text { 3. Total } \\
\text { lorazepam } \\
\text { doses (Day 3) }\end{array}$ & $\begin{array}{l}\text { Median } 2 \mathrm{mg} \\
\text { (IQR } 0 \text { to } 30 \text { ) }\end{array}$ & $\begin{array}{l}\text { Median } 2 \mathrm{mg} \\
\text { (IQR } 0 \text { to } 6 \text { ) }\end{array}$ & \\
\hline & & & & 4. ED discharge & $4 / 40(10.0 \%)$ & 2/38 (5.3\%) & \\
\hline & & & & $\begin{array}{l}\text { 5. Hospital } \\
\text { admission (non- } \\
\text { ICU) }\end{array}$ & $\begin{array}{l}34 / 40 \\
(85.0 \%)\end{array}$ & $32 / 38(84.2 \%)$ & \\
\hline & & & & $\begin{array}{l}\text { 6. ICU } \\
\text { admission }\end{array}$ & $2 / 40(5.0 \%)$ & 4/38 (10.5\%) & \\
\hline & & & & $\begin{array}{l}\text { 7. Discharged } \\
\text { within three } \\
\text { days of } \\
\text { admission }\end{array}$ & $9 / 40(22.5 \%)$ & 2/38 (5.3\%) & \\
\hline \multirow{6}{*}{$\begin{array}{l}\text { Sullivan SM, } \\
\text { Dewey BN, } \\
\text { Jarrell DJ, } \\
\text { Vadiei N, } \\
\text { Patanwala AE } \\
\text { (2019) [34] }\end{array}$} & \multirow{6}{*}{$\begin{array}{l}\text { Adults with primary } \\
\text { ED diagnosis of } \\
\text { AWS }\end{array}$} & \multirow{6}{*}{$\begin{array}{l}\text { Phenobarbital }+/- \\
\text { symptom-triggered } \\
\text { benzodiazepine } \\
(n=97)\end{array}$} & \multirow{6}{*}{$\begin{array}{l}\text { Symptom- } \\
\text { triggered } \\
\text { benzodiazepine } \\
(n=112)\end{array}$} & $\begin{array}{l}\text { 1. ICU } \\
\text { admission }\end{array}$ & $\begin{array}{l}14 / 97 \\
(14.4 \%)\end{array}$ & $12 / 112(10.7 \%)$ & Serious \\
\hline & & & & $\begin{array}{l}\text { 2. ED length of } \\
\text { stay }\end{array}$ & $\begin{array}{l}\text { Median } 9 \mathrm{~h} \\
\text { (IQR } 6 \text { to } 14 \text { ) }\end{array}$ & $\begin{array}{l}\text { Median } 9 \mathrm{~h} \\
\text { (IQR } 6 \text { to } 14 \text { ) }\end{array}$ & \\
\hline & & & & $\begin{array}{l}\text { 3. Median } \\
\text { hospital length } \\
\text { of stay }\end{array}$ & $\begin{array}{l}3 \text { days (IQR } 2 \\
\text { to } 5 \text { ) }\end{array}$ & 4 days (IQR 2 to 6 ) & \\
\hline & & & & $\begin{array}{l}\text { 4. Hospital } \\
\text { admission (non- } \\
\text { ICU) }\end{array}$ & $\begin{array}{l}41 / 97 \\
(42.3 \%)\end{array}$ & 60/112 (53.6\%) & \\
\hline & & & & 5. ED discharge & $\begin{array}{l}42 / 97 \\
(43.3 \%)\end{array}$ & 40/112 (35.7\%) & \\
\hline & & & & $\begin{array}{l}\text { 6. CIWA scores } \\
\text { at ED discharge }\end{array}$ & $\begin{array}{l}\text { Median } 7 \\
\text { (IQR } 4 \text { to 12) }\end{array}$ & $\begin{array}{l}\text { Median } 7 \\
\text { (IQR } 4 \text { to } 14 \text { ) }\end{array}$ & \\
\hline \multirow{5}{*}{$\begin{array}{l}\text { Rosenson J, } \\
\text { Clements C, } \\
\text { Simon B, } \\
\text { Vieaux J, } \\
\text { Graffman S, } \\
\text { Vahidnia F, } \\
\text { et al. (2013) } \\
\text { [35] }\end{array}$} & \multirow[t]{5}{*}{$\begin{array}{l}\text { Adults with } \\
\text { suspected AWS } \\
\text { (clinician } \\
\text { assessment) }\end{array}$} & \multirow[t]{5}{*}{$\begin{array}{l}\text { Phenobarbital } 10 \\
\text { mg/kg IV over } 30 \\
\text { min + symptom- } \\
\text { triggered } \\
\text { lorazepam PO/IV } \\
(\mathrm{n}=100)\end{array}$} & \multirow[t]{5}{*}{$\begin{array}{l}\text { Symptom- } \\
\text { triggered } \\
\text { lorazepam PO/IV } \\
(n=98)\end{array}$} & $\begin{array}{l}\text { 1. ICU } \\
\text { admission }\end{array}$ & $\begin{array}{l}4 / 51 \\
(7.8 \%) \text { effect } \\
\text { size: } 17 \\
(95 \% \text { Cl } 4 \text { to } \\
32 \text { ) }\end{array}$ & 13/51 (25.5\%) & \multirow[t]{5}{*}{ High } \\
\hline & & & & $\begin{array}{l}\text { 2. Telemetry } \\
\text { unit admission }\end{array}$ & $\begin{array}{l}23 / 51 \\
(45.1 \%) \text { effect } \\
\text { size: }-6 \\
(95 \% \mathrm{Cl}-25 \\
\text { to } 13)\end{array}$ & 20/51 (39.2\%) & \\
\hline & & & & $\begin{array}{l}\text { 3. General ward } \\
\text { admission }\end{array}$ & $\begin{array}{l}24 / 51 \\
(47.1 \%) \text { effect } \\
\text { size: }-12 \\
(95 \% \mathrm{Cl}-31 \\
\text { to } 7)\end{array}$ & 18/51 (35.3\%) & \\
\hline & & & & $\begin{array}{l}\text { 4. Hospital } \\
\text { length of stay } \\
\text { (non-ICU) }\end{array}$ & $\begin{array}{l}\text { Median } 76 \mathrm{~h} \\
\text { (IQR } 54 \text { to } \\
\text { 114)effect } \\
\text { size: } 42 \\
(95 \% \mathrm{Cl}-4 \\
\text { to } 82 \text { ) }\end{array}$ & $\begin{array}{l}\text { Median } 118 \text { h (IQR } 47 \\
\text { to 190) }\end{array}$ & \\
\hline & & & & $\begin{array}{l}\text { 5. ICU length of } \\
\text { stay }\end{array}$ & $\begin{array}{l}\text { Median } 34 \mathrm{~h} \\
\text { (IQR } 30 \text { to } \\
\text { 276) effect } \\
\text { size: } 60(95 \% \\
\mathrm{Cl}-170 \text { to } \\
434)\end{array}$ & $\begin{array}{l}\text { Median } 94 \mathrm{~h} \text { (IQR } 43 \\
\text { to } 134 \text { ) }\end{array}$ & \\
\hline
\end{tabular}


Table 2 Main outcomes, key findings, and risk of bias assessments for summarized studies (Continued)

\begin{tabular}{|c|c|c|c|c|c|c|c|}
\hline Author & Participants & Intervention & Comparison & $\begin{array}{l}\text { Main } \\
\text { outcome(s) }\end{array}$ & Key results & & $\begin{array}{l}\text { Risk of } \\
\text { bias }\end{array}$ \\
\hline \multicolumn{8}{|c|}{ Phenobarbital alone } \\
\hline \multirow{3}{*}{$\begin{array}{l}\text { Nelson AC, } \\
\text { Kehoe J, } \\
\text { Sankoff J, } \\
\text { Mintzer D, } \\
\text { Taub J, } \\
\text { Kaucher KA } \\
\text { (2019) [36] }\end{array}$} & \multirow[t]{3}{*}{$\begin{array}{l}\text { Adults requiring } \\
\text { medical treatment } \\
\text { for AWS (clinician } \\
\text { assessment) }\end{array}$} & \multirow[t]{3}{*}{$\begin{array}{l}\text { Phenobarbital IV } \\
(n=100)\end{array}$} & \multirow[t]{3}{*}{$\begin{array}{l}\text { 1. Diazepam IV } \\
(n=100) 2 . \\
\text { Phenobarbital } \\
\text { IV + lorazepam IV } \\
(n=100)\end{array}$} & $\begin{array}{l}\text { 1. ICU } \\
\text { admission }\end{array}$ & $\begin{array}{l}13 / 100 \\
(13.0 \%)\end{array}$ & $\begin{array}{l}\text { Diazepam: 8/100 } \\
\text { (8.0\%)Phenobarbital } \\
\text { + lorazepam: } 11 / 100 \\
\text { (11.0\%) }\end{array}$ & \multirow[t]{3}{*}{ Serious } \\
\hline & & & & $\begin{array}{l}\text { 2. Hospital } \\
\text { admission (non- } \\
\text { ICU) }\end{array}$ & $\begin{array}{l}41 / 100 \\
(41.0 \%)\end{array}$ & $\begin{array}{l}\text { Diazepam: } 27 / 100 \\
\text { (27.0\%)Phenobarbital } \\
\text { + lorazepam: } 36 / 100 \\
(36.0 \%)\end{array}$ & \\
\hline & & & & $\begin{array}{l}\text { 3. Hospital } \\
\text { length of stay } \\
\text { (non-ICU) }\end{array}$ & $96 \mathrm{~h}$ & $\begin{array}{l}\text { Diazepam: } 137 \\
\text { hPhenobarbital + } \\
\text { lorazepam: } 71 \mathrm{~h}\end{array}$ & \\
\hline \multirow[t]{3}{*}{$\begin{array}{l}\text { Hendey GW, } \\
\text { Dery RA, } \\
\text { Barnes RL, } \\
\text { Snowden B, } \\
\text { Mentler P } \\
\text { (2011) [42] }\end{array}$} & \multirow[t]{3}{*}{$\begin{array}{l}\text { Adults with known } \\
\text { or suspected AWS } \\
\text { (clinician } \\
\text { assessment) }\end{array}$} & \multirow{3}{*}{$\begin{array}{l}\text { Phenobarbital } 260 \\
\text { mg IV (initial } \\
\text { dose) }+130 \text { mg IV } \\
\text { (subsequent doses) } \\
\text { repeated at } \\
\text { physician discretion } \\
(n=25)\end{array}$} & \multirow{3}{*}{$\begin{array}{l}\text { Lorazepam } 2 \mathrm{mg} \\
\text { IV (initial dose) + } \\
2 \mathrm{mg} \mathrm{IV} \\
\text { (subsequent } \\
\text { doses) repeated } \\
\text { at physician } \\
\text { discretion } \\
(n=19)\end{array}$} & $\begin{array}{l}\text { 1. Change in } \\
\text { CIWA score } \\
\text { (from baseline } \\
\text { to ED } \\
\text { discharge) }\end{array}$ & -9.6 & -12.6 & \multirow[t]{3}{*}{$\begin{array}{l}\text { Some } \\
\text { concerns }\end{array}$} \\
\hline & & & & $\begin{array}{l}\text { 2. ED length of } \\
\text { stay }\end{array}$ & $267 \mathrm{~min}$ & $256 \min$ & \\
\hline & & & & $\begin{array}{l}\text { 3. Hospital } \\
\text { admission }\end{array}$ & $\begin{array}{l}12 / 25 \\
(48.0 \%)\end{array}$ & 16/19 (84.2\%) & \\
\hline \multicolumn{8}{|l|}{ Phenytoin alone } \\
\hline $\begin{array}{l}\text { Rathlev NK, } \\
\text { D'Onofrio G, } \\
\text { Fish SS, } \\
\text { Harrison PM, } \\
\text { Bernstein E, } \\
\text { Hossack RW, } \\
\text { et al. (1994) } \\
\text { [37] }\end{array}$ & $\begin{array}{l}\text { Adults with alcohol } \\
\text { withdrawal seizure }\end{array}$ & $\begin{array}{l}\text { Phenytoin } 15 \mathrm{mg} / \\
\mathrm{kg} \text { IV over } 20 \mathrm{~min} \\
(n=49)\end{array}$ & $\begin{array}{l}\text { Normal saline } \\
\text { placebo }(n=51)\end{array}$ & $\begin{array}{l}\text { Post-infusion } \\
\text { seizure } \\
\text { recurrence } \\
\text { within } 6 \mathrm{~h}\end{array}$ & $\begin{array}{l}10 / 49 \\
(20.4 \%) \text { effect } \\
\text { size: } 3 \\
(95 \% \mathrm{Cl}:-16 \\
\text { to } 16)\end{array}$ & $12 / 51(23.5 \%)$ & Low \\
\hline $\begin{array}{l}\text { Chance JF } \\
\text { (1991) [43] }\end{array}$ & $\begin{array}{l}\text { Adults with alcohol } \\
\text { withdrawal seizure }\end{array}$ & $\begin{array}{l}\text { Phenytoin } 15 \text { mg/ } \\
\text { kg IV (maximum } \\
\text { dose } 1000 \text { mg, } \\
\text { maximum rate } 37 \\
\text { mg/min) }(n=28)\end{array}$ & $\begin{array}{l}\text { Normal saline } \\
\text { placebo }(n=27)\end{array}$ & $\begin{array}{l}\text { Post-infusion } \\
\text { seizure } \\
\text { recurrence } \\
\text { within } 6 \mathrm{~h}\end{array}$ & $\begin{array}{l}6 / 28 \\
(21.4 \%) \text { effect } \\
\text { size: } 2 \\
(95 \% \mathrm{Cl}:-20 \\
\text { to } 16)\end{array}$ & 5/27 (18.5\%) & Low \\
\hline $\begin{array}{l}\text { Alldredge BK, } \\
\text { Lowenstein } \\
\text { DH, Simon RP } \\
\text { (1989) [38] }\end{array}$ & $\begin{array}{l}\text { Adults with alcohol } \\
\text { withdrawal seizure }\end{array}$ & $\begin{array}{l}\text { Phenytoin } 1000 \mathrm{mg} \\
\text { IV over } 20 \mathrm{~min}(n= \\
45)\end{array}$ & $\begin{array}{l}\text { Normal saline } \\
\text { placebo }(n=45)\end{array}$ & $\begin{array}{l}\text { Post-infusion } \\
\text { seizure } \\
\text { recurrence } \\
\text { within } 12 \mathrm{~h}\end{array}$ & $\begin{array}{l}6 / 45 \\
(13.3 \%) \text { effect } \\
\text { size: } 0 \\
(95 \% \mathrm{Cl}:-14 \\
\text { to } 14)\end{array}$ & 6/45 (13.3\%) & $\begin{array}{l}\text { Some } \\
\text { concerns }\end{array}$ \\
\hline
\end{tabular}

* statistically significant difference between groups $(p<0.05)$

AWS $=$ alcohol withdrawal syndrome

CIWA = Clinical Institute Withdrawal Assessment for Alcohol

$\mathrm{ED}=$ emergency department

$\mathrm{ICU}=$ intensive care unit

IV = intravenous

\section{Phenobarbital alone}

One retrospective cohort study $(n=300)$ compared phenobarbital alone with two different protocols: 1) IV diazepam alone, and 2) combined IV phenobarbital and IV lorazepam [36]. The three protocols were performed in different time periods and were the result of medication shortages. There were no differences between the three groups in the primary outcome of ICU admission (13.0, 8.0, and $11.0 \%$ respectively, $p=0.99)$.

Only one RCT $(n=44)$ compared phenobarbital alone versus benzodiazepines [42]. Patients in the phenobarbital group received an initial $260 \mathrm{mg}$ IV dose, and subsequent $130 \mathrm{mg}$ IV doses repeated at physicians' discretion. The comparison group received an initial lorazepam $2 \mathrm{mg}$ IV dose, and subsequent 2 mg IV doses repeated at physicians' discretion. Phenobarbital and lorazepam were similarly effective in treating mild or moderate alcohol withdrawal in the ED (no significant difference in change in CIWA scores between groups), with similar ED length of stay (267 $\mathrm{min}$ vs. $256 \mathrm{~min}, p=0.8$ ) and hospital admissions (48.0\% vs. $84.2 \%, p=0.8$ ) [42]. 


\section{Phenytoin alone}

Three RCTs published before 1995 found no significant benefit to phenytoin compared to normal saline placebo in preventing seizure recurrence in the ED. [37, 38, 43]

\section{Discussion}

\section{Summary of evidence}

After an initial generalized seizure resulting from alcohol withdrawal, a single dose of IV lorazepam prevented seizure recurrence in the ED. [31] Sublingual lorazepam is more effective compared to placebo in reducing CIWA scores among patients in mild-to-moderate withdrawal [39]. There is no clear evidence that any one benzodiazepine is superior to another at improving withdrawal symptoms or preventing complications related to alcohol withdrawal syndrome [32].

Symptom-triggered protocols have been implemented in EDs with clinical decision units that can support longer stays, although patient outcomes have not been rigorously evaluated $[33,40]$. One retrospective chart review suggested that a symptom-triggered protocol may decrease total doses of benzodiazepines administered, however, this finding would need to be replicated in a prospective, controlled study [40].

Existing studies do not show uniform evidence of benefit of phenobarbital (used alone, or in conjunction with symptom-triggered benzodiazepines) in multiple assessed outcomes: ICU admission, ED length of stay, and complications such as intubation [34-36, 41]. One RCT showed a $17.0 \%$ lower ICU admission (95\% CI: 4.0 to $32.0 \%$ ) among patients treated with IV phenobarbital combined with symptom-triggered lorazepam, but we assessed this study to be at high risk of bias [35].

Phenytoin is not effective versus normal saline placebo at preventing seizure recurrence related to alcohol withdrawal syndrome [37, 38, 43].

\section{Limitations}

This review is limited by the overall poor quality of included studies, most of which were at high/serious risk of bias. We identified a lack of standardized definitions of alcohol withdrawal syndrome and severity among included studies. Studies also poorly reported detailed inclusion criteria, and/or clinical/patient information that would allow an interpretation of the populations most likely to benefit from each type of intervention.

Our use of rapid review methodology may increase the chance of inaccuracies in our study assessments vis-à-vis a formal systematic review. Nonetheless, we employed a systematic search strategy and our trained reviewers applied rigorous, prespecified criteria for inclusion, extraction, and risk of bias assessments, which strengthen our approach. Furthermore, our findings contribute more rigorous evidence compared to those previously published in expert opinion articles and narrative reviews. As most included studies were conducted in the United States and Canada, we are confident that our findings are likely generalizable within the North American context.

\section{Conclusions}

\section{Comparison to previous studies}

Our review highlights a paucity of studies evaluating the safety and efficacy of guideline-supported treatments for alcohol withdrawal syndrome (e.g., gabapentin and clonidine) when provided specifically in the ED setting $[15,16]$.

Unlike in the outpatient setting, ED patients generally present with more severe manifestations of withdrawal and are likely more medically complex. However, they may be more easily monitored, and medications and supportive treatments can be administered intravenously. Unlike in the inpatient setting, ED patients have undifferentiated presentations, are often being managed in high-volume settings (where care spaces, time for assessments, and clinical resources are stretched), and typically do not remain in the ED for more than $24 \mathrm{~h}$. After ED treatment, clinicians must determine if patients are safe for discharge, or if they require hospital admission for further management.

Given the key differences between the ED compared to outpatient and inpatient contexts, there is a need for rigorous evidence evaluating the safety and effectiveness of ED-specific treatment approaches, and further guidance for risk stratification and resource allocation.

Finally, a previous review by Long et al. (2017), which summarized evidence from non-ED settings and noninterventional studies, proposed an algorithmic approach to alcohol withdrawal syndrome in the ED consisting of escalating doses of benzodiazepines, followed by phenobarbital, then propofol [20]. Our review supports the use of benzodiazepines as first-line treatment of severe alcohol withdrawal in the ED. However, our review of evidence from interventional studies performed in the ED does not provide sufficient evidence to recommend routine use of phenobarbital or propofol in ED treatment algorithms.

\section{Clinical implications}

Benzodiazepines are the most evidence-based treatment for alcohol withdrawal treatment in the ED, especially for the prevention of alcohol withdrawal seizure recurrence. However, no clear evidence supports the use of one type of benzodiazepine over others. It is unclear if symptom-triggered protocols are effective for use in EDs, especially in those without attached observational units that can support longer stays. More evidence is needed to determine if phenobarbital, whether in combination with benzodiazepines or used alone, can be 
used safely and effectively for treatment of alcohol withdrawal syndrome in the ED, especially with regards to dosing, timing, and need for hospital admission. Phenytoin does not have evidence of effectiveness at preventing alcohol withdrawal seizures in the ED.

\section{Research implications}

Given the rapidly changing landscape of alcohol-related ED visits during the COVID-19 pandemic, and the potential for new treatment strategies to quickly emerge, there is an urgent need in the near future for a full systematic review and evidence synthesis. Future studies should standardize definitions of alcohol withdrawal, outcome measures, and ascertainment of outcomes and adverse events; and distinguish between EDs with and without attached observational units; such that they can generate rigorous and generalizable evidence to guide ED management. Further studies are needed to evaluate symptom-triggered benzodiazepine protocols in the ED. Pharmacotherapies that have demonstrated benefit for treatment of alcohol withdrawal in other settings need to be evaluated in the ED setting before routine use.

\section{Appendix A Search strategy for MEDLINE (Ovid) \\ Search Results51 49 and 50 [Clinical Tri- als] (14) 5452 and 53 [Observ] (37) 5855 not 57 [Remaining] (35) Date: May 11, 2020Database: Ovid MEDLINE(R) and Epub Ahead of Print, In-Process \& Other Non- Indexed Citations, Daily and Versions (R) < 1946 to May 08, 2020>Search Strategy:-----}

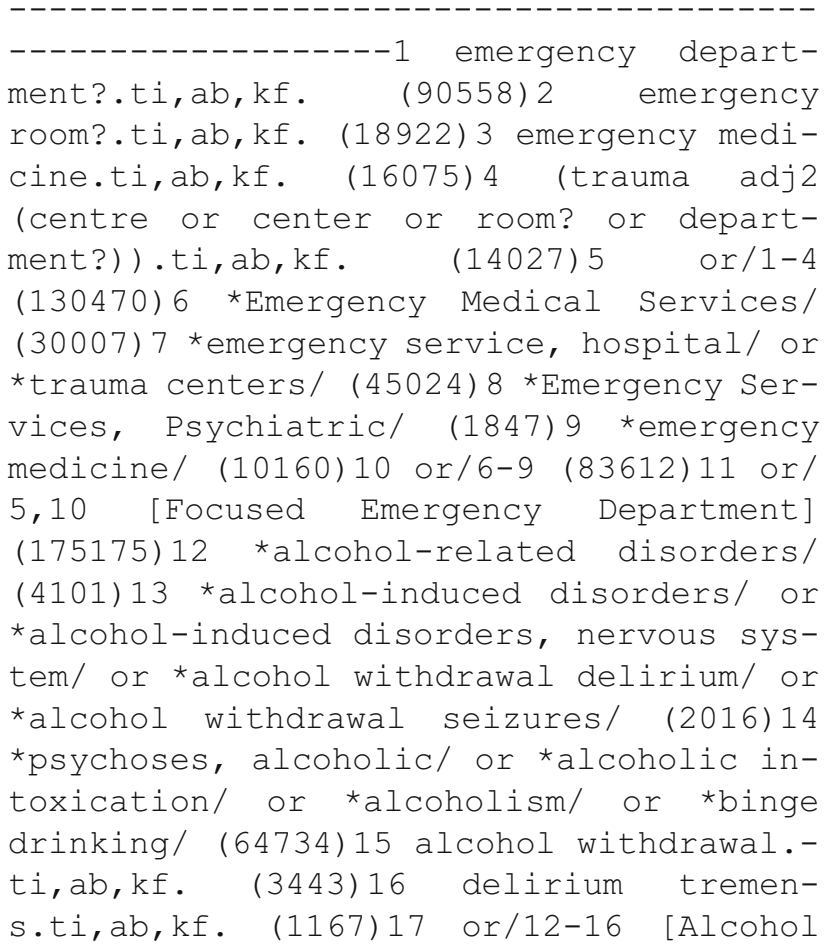

Withdrawal] (71222) 1811 and 17 [ED \& AlCohol Withdrawal] (1422)19 exp benzodiazepines/ (65052) 20 Benzodiazepine?.ti,ab, kw. (34309)21 exp barbiturates/ (53623)22 exp adrenergic alpha-2 receptor agonists/ (25402)23 exp "hypnotics and sedatives"/ (122536) 24 exp antipsychotic agents/ (123243) 25 exp Anticonvulsants/ (140816)26 baclofen/ (5580) 27 Ethanol/tu, th [Therapeutic Use, Therapy] (2808) 28 or/19-27 [Drug Therapy] (387290) 29 and $/ 11,17,28$ [ED \& Alcohol Disorders \& Drug Therapy] (86) $30 \mathrm{dt}$.fs. [Drug Therapy] (2201520) 31 ad.fs. [Administration \& Dose] (1402502)32 tu.fs. [Therapeutic Use] (2205294)33 Drug Therapy, Combination/ (164791)34 Dose-Response Relationship, Drug/ (401281)35 de.fs. [Drug Effects] (2966175) 36 Drug Interactions/ (84883) 37 or/30-36 [Pharmacological therapy] (5758470)38 and/11,17,37 [ED \& Alcohol Disorders \& Pharmacological therapy] (126) 3911 and 17 and (28 or 37) [ED \& Alcohol Disorders \& Clinical Intervention] (159) 40 limit 39 to yr="1980 -Current" (157) 41 limit 40 to English language (148) 42 comment/ or editorial/ or letter/ or news/ (2022920)4341 not 42 (141) 44 animals/ not (animals/ and humans/) (4663791) 4543 not 44 (139) 46 limit 45 to (systematic reviews pre 2019 or systematic reviews) (10) 47 limit 45 to "reviews (best balance of sensitivity and specificity)" (26) 48 or/46-47 [Reviews] (29) 4945 not 48 [Remaining] (110)50 exp clinical trial/ (858383)51 49 and 50 [Clinical Trials] (14) 5249 not 51 [Remaining] (96) 53 retrospective studies/ or cohort studies/ or follow-up studies/ or prospective studies/ or controlled before-after studies/ or cross-sectional studies/ or Comparative Study/ (3689327)54 52 and 53 (37) 5552 not 54 [Remaining] (59)56 case reports/ (2095639) $57 \quad 55$ and 56 (24) $58 \quad 55$ not 57 [Remaining] (35)

\section{Acknowledgements}

None.

\section{Authors' contributions}

The investigators controlled all aspects of data collection, analysis, and report writing. JM and MDW conceptualized and drafted the protocol. MM, JK, and JM screened abstracts and articles for inclusion. JK assessed article quality. MDW developed and ran the search strategy. JK and MM drafted the manuscript and all authors contributed to its revision. JK takes overall responsibility for the study. All authors read and approved the final manuscript. 


\section{Funding}

This study was unfunded. This research received no specific grant from any funding agency, commercial, or not-for-profit sectors.

\section{Availability of data and materials}

All data analyzed during this study are included in the published article.

\section{Declarations}

Ethics approval and consent to participate

Not applicable.

\section{Consent for publication}

Not applicable.

\section{Competing interests}

The authors declare that they have no competing interests.

\section{Author details}

${ }^{1}$ Addiction Medicine Fellowship Program, British Columbia Centre for Substance Use, Vancouver, BC, Canada. ${ }^{2}$ Royal College Emergency Medicine Residency Program, Department of Emergency Medicine, College of Medicine, University of Saskatchewan, Saskatoon, SK, Canada. ${ }^{3}$ Faculty of Medicine, University of British Columbia, Vancouver, BC, Canada. ${ }^{4}$ Centre for Clinical Epidemiology and Evaluation, Vancouver Coastal Health Research Institute, Vancouver, BC, Canada. ${ }^{5}$ Department of Emergency Medicine, University of British Columbia, Vancouver, BC, Canada. ${ }^{6}$ Department of Emergency Medicine, Vancouver General Hospital, Vancouver, BC, Canada. British Columbia Centre for Disease Control, Vancouver, BC, Canada.

Received: 14 February 2021 Accepted: 22 October 2021

Published online: 06 November 2021

\section{References}

1. Urbanoski K, Cheng J, Rehm J, Kurdyak P. Frequent use of emergency departments for mental and substance use disorders. Emerg Med J. 2018; 35(4):220-5. https://doi.org/10.1136/emermed-2015-205554

2. Brubacher JR, Mabie A, Ngo M, Abu-Laban RB, Buchanan J, Shenton T, et al. Substance-related problems in patients visiting an urban Canadian emergency department. CJEM. 2008;10(3):198-204. https://doi.org/10.1017/ S1481803500010101

3. Myran DT, Hsu AT, Smith G, Tanuseputro P. Rates of emergency department visits attributable to alcohol use in Ontario from 2003 to 2016: a retrospective population-level study. CMAJ. 2019;191(29):E804-E10. https:// doi.org/10.1503/cmaj.181575.

4. White AM, Slater ME, Ng G, Hingson R, Breslow R. Trends in alcohol-related emergency department visits in the United States: results from the Nationwide emergency department sample, 2006 to 2014. Alcohol Clin Exp Res. 2018;42(2):352-9. https://doi.org/10.1111/acer.13559.

5. Collaborators GBDA. Alcohol use and burden for 195 countries and territories, 1990-2016: a systematic analysis for the global burden of disease study 2016. Lancet. 2018;392(10152):1015-35. https://doi.org/10.1016/S01406736(18)31310-2

6. Wood E, Albarqouni L, Tkachuk S, Green CJ, Ahamad K, Nolan S, et al. Will this hospitalized patient develop severe alcohol withdrawal syndrome?: the rational clinical examination systematic review. JAMA. 2018;320(8):825-33. https://doi.org/10.1001/jama.2018.10574.

7. Wolf C, Curry A, Nacht J, Simpson SA. Management of Alcohol Withdrawal in the emergency department: current perspectives. Open Access Emerg Med. 2020;12:53-65. https://doi.org/10.2147/OAEM.S235288.

8. Stehman CR, Mycyk MB. A rational approach to the treatment of alcohol withdrawal in the ED. Am J Emerg Med. 2013;31(4):734-42. https://doi.org/1 0.1016/j.ajem.2012.12.029

9. Klein LR, Cole JB, Driver BE, Battista C, Jelinek R, Martel ML. Unsuspected critical illness among emergency department patients presenting for acute alcohol intoxication. Ann Emerg Med. 2018;71(3):279-88. https://doi.org/10.1 016/j.annemergmed.2017.07.021.

10. Hulme J, Sheikh H, Xie E, Gatov E, Nagamuthu C, Kurdyak P. Mortality among patients with frequent emergency department use for alcoholrelated reasons in Ontario: a population-based cohort study. CMAJ. 2020; 192(47):E1522-E31. https://doi.org/10.1503/cmaj.191730.
11. Schimmel J, Vargas-Torres C, Genes N, Probst MA, Manini AF. Changes in alcohol-related hospital visits during COVID-19 in new York City. Addiction. 2021. https://doi.org/10.1111/add.15589.

12. Sharma RA, Subedi K, Gbadebo BM, Wilson B, Jurkovitz C, Horton T. Alcohol withdrawal rates in hospitalized patients during the COVID-19 pandemic. JAMA Netw Open. 2021;4(3):e210422. https://doi.org/10.1001/jama networkopen.2021.0422.

13. Narasimha VL, Shukla L, Mukherjee D, Menon J, Huddar S, Panda UK, et al. Complicated alcohol withdrawal-an unintended consequence of COVID-19 lockdown. Alcohol Alcohol. 2020;55(4):350-3. https://doi.org/10.1093/alcalc/a gaa042.

14. Smalley CM, Malone DA Jr, Meldon SW, Borden BL, Simon EL, Muir MR, et al. The impact of COVID-19 on suicidal ideation and alcohol presentations to emergency departments in a large healthcare system. Am J Emerg Med. 2021;41:237-8. https://doi.org/10.1016/j.ajem.2020.05.093.

15. British Columbia Centre on Substance Use (BCCSU). Provincial Guideline for the Clinical Management of High-Risk Drinking and Alcohol Use Disorder. Vancouver, BC: BCCSU; 20192019 December.

16. Alcohol Use Disorder Among Older Adults Guideline Development Working Group. Canadian Guidelines on Alcohol Use Disorder Among Older Adults. Canadian Coalition for Seniors' Mental Health, 20192019.

17. National Institute for Health and Care Excellence. Acute alcohol withdrawal 2020 [updated Oct 30, 2020; cited 2021 Feb 8, 2021]. Available from: http:// pathways.nice.org.uk/pathways/alcohol-use-disorders.

18. World Health Organization. Management of alcohol withdrawal: World Health Organization; 2012 [cited 2021 Feb 8, 2021]. Available from: https:// www.who.int/mental_health/mhgap/evidence/alcohol/q2/en/.

19. Kumar D, Argáez C. Gabapentin, Phenobarbital, Diazepam, and Lorazepam for the treatment of alcohol withdrawal: clinical effectiveness and guidelines. Ottawa, ON: CADTH; 2020.

20. Long D, Long B, Koyfman A. The emergency medicine management of severe alcohol withdrawal. Am J Emerg Med. 2017;35(7):1005-11. https:// doi.org/10.1016/j.ajem.2017.02.002.

21. Ganann R, Ciliska D, Thomas H. Expediting systematic reviews: methods and implications of rapid reviews. Implement Sci. 2010;5(1):56. https://doi.org/1 $0.1186 / 1748-5908-5-56$

22. Khangura S, Konnyu K, Cushman R, Grimshaw J, Moher D. Evidence summaries: the evolution of a rapid review approach. Syst Rev. 2012;1(1):10. https://doi.org/10.1186/2046-4053-1-10.

23. Tricco AC, Antony J, Zarin W, Strifler L, Ghassemi M, Ivory J, et al. A scoping review of rapid review methods. BMC Med. 2015;13(1):224. https://doi.org/1 0.1186/s12916-015-0465-6

24. Hamel C, Michaud A, Thuku M, Skidmore B, Stevens A, Nussbaumer-Streit B, et al. Defining rapid reviews: a systematic scoping review and thematic analysis of definitions and defining characteristics of rapid reviews. J Clin Epidemiol. 2020;129:74-85. https://doi.org/10.1016/j.jclinepi.2020.09.041.

25. Cochrane Rapid Reviews Methods Group. About Us: The Cochrane Group; 2020 [cited 2020 Nov 16]. Available from: https://methods.cochrane.org/ra pidreviews/welcome.

26. Tricco AC, Langlois EV, Straus SE, editors. Rapid reviews to strengthen health policy and systems: a practical guide. Geneva: World Health Organization; 2017.

27. Khangura S, Polisena J, Clifford TJ, Farrah K, Kamel C. Rapid review: an emerging approach to evidence synthesis in health technology assessment. Int J Technol Assess Health Care. 2014;30(1):20-7. https://doi.org/10.1017/ S0266462313000664

28. Moher D, Liberati A, Tetzlaff J, Altman DG, Group P. Preferred reporting items for systematic reviews and meta-analyses: the PRISMA statement. PLoS Med. 2009;6(7):e1000097. https://doi.org/10.1371/journal.pmed.1000097.

29. Sterne JAC, Savovic J, Page MJ, Elbers RG, Blencowe NS, Boutron I, et al. RoB 2: a revised tool for assessing risk of bias in randomised trials. BMJ. 2019;366 |4898. https://doi.org/10.1136/bmj.|4898.

30. Sterne JA, Hernan MA, Reeves BC, Savovic J, Berkman ND, Viswanathan M, et al. ROBINS-l: a tool for assessing risk of bias in non-randomised studies of interventions. BMJ. 2016;355:14919. https://doi.org/10.1136/bmj.i4919.

31. D'Onofrio G, Rathlev NK, Ulrich AS, Fish SS, Freedland ES. Lorazepam for the prevention of recurrent seizures related to alcohol. N Engl J Med. 1999; 340(12):915-9. https://doi.org/10.1056/NEJM199903253401203.

32. Scheuermeyer FX, Milees I, Lane DJ, Grunau B, Grafstein E, Sljivic I, et al. Lorazepam Versus Diazepam in the Management of Emergency Department Patients With Alcohol Withdrawal. Ann Emerg Med. 2020. 
33. Ismail MF, Doherty K, Bradshaw P, O'Sullivan I, Cassidy EM. Symptomtriggered therapy for assessment and management of alcohol withdrawal syndrome in the emergency department short-stay clinical decision unit. Emerg Med J. 2019;36(1):18-21. https://doi.org/10.1136/emermed-2017-2 06997.

34. Sullivan SM, Dewey BN, Jarrell DH, Vadiei N, Patanwala AE. Comparison of phenobarbital-adjunct versus benzodiazepine-only approach for alcohol withdrawal syndrome in the ED. Am J Emerg Med. 2019;37(7):1313-6. https://doi.org/10.1016/j.ajem.2018.10.007.

35. Rosenson J, Clements C, Simon B, Vieaux J, Graffman S, Vahidnia F, et al. Phenobarbital for acute alcohol withdrawal: a prospective randomized double-blind placebo-controlled study. J Emerg Med. 2013;44(3):592-8 e2. https://doi.org/10.1016/j.jemermed.2012.07.056

36. Nelson AC, Kehoe J, Sankoff J, Mintzer D, Taub J, Kaucher KA. Benzodiazepines vs barbiturates for alcohol withdrawal: analysis of 3 different treatment protocols. Am J Emerg Med. 2019;37(4):733-6. https:// doi.org/10.1016/..ajem.2019.01.002.

37. Rathlev NK, D'Onofrio G, Fish SS, Harrison PM, Bernstein E, Hossack RW, et al. The lack of efficacy of phenytoin in the prevention of recurrent alcoholrelated seizures. Ann Emerg Med. 1994;23(3):513-8. https://doi.org/10.1016/ S0196-0644(94)70070-2.

38. Alldredge BK, Lowenstein DH, Simon RP. Placebo-controlled trial of intravenous diphenylhydantoin for short-term treatment of alcohol withdrawal seizures. Am J Med. 1989;87(6):645-8. https://doi.org/10.1016/ 50002-9343(89)80397-3.

39. Naranjo CA, Sellers EM, Chater K, Iversen P, Roach C, Sykora K Nonpharmacologic intervention in acute alcohol withdrawal. Clin Pharmacol Ther. 1983;34(2):214-9. https://doi.org/10.1038/clpt.1983.155.

40. Cassidy EM, O'Sullivan I, Bradshaw P, Islam T, Onovo C. Symptom-triggered benzodiazepine therapy for alcohol withdrawal syndrome in the emergency department: a comparison with the standard fixed dose benzodiazepine regimen. Emerg Med J. 2012;29(10):802-4. https://doi.org/10.1136/ emermed-2011-200509.

41. Ibarra F Jr. Single dose phenobarbital in addition to symptom-triggered lorazepam in alcohol withdrawal. Am J Emerg Med. 2020;38(2):178-81. https://doi.org/10.1016/j.ajem.2019.01.053.

42. Hendey GW, Dery RA, Barnes RL, Snowden B, Mentler P. A prospective, randomized, trial of phenobarbital versus benzodiazepines for acute alcohol withdrawal. Am J Emerg Med. 2011;29(4):382-5. https://doi.org/10.1016/j.a jem.2009.10.010

43. Chance JF. Emergency department treatment of alcohol withdrawal seizures with phenytoin. Ann Emerg Med. 1991;20(5):520-2. https://doi.org/10.1016/ S0196-0644(05)81606-3.

\section{Publisher's Note}

Springer Nature remains neutral with regard to jurisdictional claims in published maps and institutional affiliations.

Ready to submit your research? Choose BMC and benefit from:

- fast, convenient online submission

- thorough peer review by experienced researchers in your field

- rapid publication on acceptance

- support for research data, including large and complex data types

- gold Open Access which fosters wider collaboration and increased citations

- maximum visibility for your research: over $100 \mathrm{M}$ website views per year

At $\mathrm{BMC}$, research is always in progress.

Learn more biomedcentral.com/submissions 